\title{
Review on the Neutron Source Strength of HEU Metal Burst Reactor
}

\author{
Xiaobo Liu \\ Institute of Nuclear Physics and Chemistry, CAEP \\ No. 64 Mianshan, Mianyang, China 621900 \\ liu_express11@yahoo.com
}

\begin{abstract}
There were two similar series of burst waiting time experiments carried out on Godiva-II and Caliban respectively and analyzed for neutron initiation study, unfortunately both the strength of spontaneous fission neutron source given in the published papers are not consistent with the spontaneous fission data of U-235 and U-238.

In this paper, the discrepancies of spontaneous fission data of U-235 and U-238 from different references are reviewed and clarified and the correct strength of spontaneous fission neutron source for Godiva-II and Caliban are calculated respectively, then the multiplication equivalence method of arbitrary source is introduced and the equivalent fundamentalmode(EFM) co-efficiencies of spontaneous fission neutron source for Godiva-II and Caliban are calculated with MC software, lastly the strength of neutron source for these two HEU metal burst reactor in EFM are deduced. The EFM neutron strength of this two burst reactors are much less than proposed values used for burst waiting investigation in published papers respectively, and with the new data, the inconsistency of burst waiting time to the published results is presented and discussed.
\end{abstract}

KEYWORDS: Godiva-II; Caliban; HEU metal burst reactor; neutron initiation; spontaneous fission neutron source; equivalent fundamental-mode neutron source

\section{INTRODUCTION}

Godiva-II and Caliban are the same type of HEU metal, fast neutron burst reactors, and both can be operated to produce a burst in a slightly super prompt-critical. This makes them one kind of an excellent platform for the investigation of fission chain stochastic and neutron initiation.

There were two similar series of burst waiting time experiments carried out on Godiva-II in 1960s by T. F. WIMETT[1] and on Caliban in 1980s- but published later in 2012[2], respectively. The data were used as a sample analyzed for the investigation of neutron initiation, but the experimental results had not matched the analytical results with the Hansen initiation model[3]. This problem had remained for almost half century until Xiao resolved it in 2011[4] with the fission chain stochastic simulation method. Later in 2014 Nicolas Authier analyzed the Caliban's waiting time results with the same simulation method, and drew the same conclusion that the simulation matches the experiment well[5].

For this type of experiments, the neutron source includes two kinds of origins, one is the spontaneous fission neutron (intrinsic source) from U-235 and U-238, the other is from the delayed neutrons precursors which are born and accumulated before the burst initiation takes place. The strength of delayed neutron source is 
definitely subject to the strength of intrinsic source. This makes this type of experiment hard to be used for initiation study, however $\mathrm{Liu}[6,7]$ established a novel experimental method for validating the neutron initiation

Unfortunately both the strength of spontaneous fission neutron source used for Godiva-II and Caliban waiting time experiment simulation in the published papers are not consistent with the spontaneous fission data of U-235 and U-238. Whether and to what extent this will affect the simulation results is worthy for further investigation.

In this paper, firstly the basic information of Godiva-II and Caliban reactor and the discrepancies of spontaneous fission data of U-235 and U-238 from different references are reviewed, then the EFM coefficiencies for spontaneous fission neutron source and EFM neutron strength of these two reactors are calculated; lastly with the new neutron strength data, the waiting time experiment simulation was carried out and the inconsistency of burst waiting time between this results to the published results is presented and discussed.

\section{Godiva-II, Caliban and their Intrinsic Neutron Source}

\subsection{Godiva-II}

The Godiva II reactor was the first metal reactor designed specifically for the routine production of fission bursts and was operated initially in June, 1957. It had a cylinder core in dimension of 6.9 in. diameter and 5.7 in. high, with totally $57.7 \mathrm{~kg}$ wt $93.5 \%$ casted high enriched uranium. The 94 burst of waiting time experiment was carried out and published in 1960 at a reactivity above prompt critical of $0.05 \$$, and the results of the mean initiation time was $3.1 \mathrm{~s} \pm 2.5 \mathrm{~s}$.[1]

Regarding to the strength of intrinsic source, $90 \mathrm{~s}^{-1}$ was given by T. F. WIMETT[1] for initiation theory study and $84.2 \mathrm{~s}^{-1}$ in EFM was given by Xiao[4] for waiting time simulation, however there was not any further information to explain how this values was obtained.

\subsection{Caliban}

The CALIBAN reactor, operated at the CEA Valduc center in France, is an unreflected highly enriched uranium (HEU) metal fast burst reactor, like the Godiva reactor in the USA. The CALIBAN reactor is cylinder shape in dimension of $25.2 \mathrm{~cm}$ diameter and $19.5 \mathrm{~cm}$ high, and made of ten fuel discs and four rods of $93.5 \%$ enriched-uranium metal alloyed with $10 \mathrm{wt} \%$ molybdenum, with a combined weight of 113 $\mathrm{kg}$. The 114 burst of waiting time experiment was carried out and published in 2012 at a reactivity above prompt critical of $0.083 \$$, and the results of the mean initiation time was $0.742 \mathrm{~s} \pm 0.700 \mathrm{~s} .[2,5]$

Regarding to the strength of intrinsic source, $200 \mathrm{~s}^{-1}$ was given by Philippe Humbert[2] and Nicolas Authier[5] for burst initiation simulation. Here quote that "we find a total rate of fission of 75.6 fissions/s unmultiplied, which leads to the rounded value of $200 \mathrm{n} / \mathrm{s}$ in everyday use." From this quotation it can be deduced that the average multiplicity for spontaneous fission (SF) used implicitly was 2.64 .

\subsection{Intrinsic Neutron Source}

Some references presented the nuclear data for spontaneous fission of U-235 and U-238, as shown Table I. 
Regarding to the average neutron multiplicity for SF of U-235 and U-238, there are almost the same values recommended by the four references in Table I, which is 1.86 and 2.07 respectively. However the average multiplicity (nubar) for the fast induced fission of U-235 and U-238 is 2.57 and 2.79 respectively (Table 6.3 in [8]), which are about $38 \%$ and $35 \%$ more than SF of U-235 and U-238.

Regarding to SF the neutron emission rates, the great discrepancy existed from one to other for the U-235.

Table I. SF Neutron Emission Rates and SF Multiplicity for U-235 and U-238

\begin{tabular}{|c|c|c|c|c|c|}
\hline & Reference & $\begin{array}{c}\mathrm{U}-235 \\
\left(\mathrm{~g}^{-1} \mathrm{~s}^{-1}\right)\end{array}$ & $\begin{array}{c}\mathrm{U}-238 \\
\left(\mathrm{~g}^{-1} \mathrm{~s}^{-1}\right)\end{array}$ & $\begin{array}{c}\text { SF Multiplicity } \\
\text { for U-235 }\end{array}$ & $\begin{array}{c}\text { SF Multiplicity } \\
\text { for U-238 }\end{array}$ \\
\hline 1 & Table 6.2 in [8], 2008 & $3.0 \times 10^{-4}$ & $1.39 \times 10^{-2}$ & 1.86 & 2.07 \\
\hline 2 & Table 5.1 in [9], 1998 & $2.99 \times 10^{-4}$ & $1.39 \times 10^{-2}$ & 1.86 & 2.01 \\
\hline 3 & TABLE I in [10], 1997 & $1.0 \times 10^{-5}$ & $1.36 \times 10^{-2}$ & 1.86 & 2.07 \\
\hline 4 & TABLE 1 in [11],1993 & $8.0 \times 10^{-4}$ & $1.60 \times 10^{-2}$ & — $^{*}$ & - $^{*}$ \\
\hline
\end{tabular}

$*$ No data available in the original reference.

If the data from the 1st entry in Table I are used for calculate the total neutron strength SF of uranium for the Godiva-II and Caliban, the strength of their intrinsic neutron source should be $68 \mathrm{~s}^{-1}$ and $121 \mathrm{~s}^{-1}$, respectively. These values are much less than proposed above respectively in the published paper, as shown in Table II.

Table II. Strength of Intrinsic Neutron Source for U-235 and U-238

\begin{tabular}{|c|c|c|c|c|c|}
\hline & $\begin{array}{c}\text { Mass of core } \\
(\mathrm{kg})\end{array}$ & $\begin{array}{c}\text { Mo content } \\
(\mathrm{wt} \%)\end{array}$ & $\begin{array}{c}\text { U-235 enrichment } \\
(\mathrm{wt} \%)\end{array}$ & $\begin{array}{c}\text { Intrinsic neutron } \\
\text { source this work } \\
\left(\mathrm{s}^{-1}\right)^{*}\end{array}$ & $\begin{array}{c}\text { Source proposed } \\
\text { by paper } \\
\left(\mathrm{s}^{-1}\right)\end{array}$ \\
\hline Godiva-II & 57.7 & 0 & $93.50 \%$ & $68(16+52)$ & 90 \\
\hline Caliban & 113 & 10 & $93.50 \%$ & $121(28.5+92)$ & 200 \\
\hline
\end{tabular}

* The SF neutron contribution from the U-234, U-236 is negligible and not accounted for.

The values in the bracket are the SF neutron contribution from the U-235 and U-238 respectively.

\subsection{Effect on the Initiation Simulation and Study}

As G. D. Spriggs pointed out in 1997[10], when doing neutron dynamic study with point model, the neutron source strength should be the EFM strength rather than of the spontaneous fission source from the fission isotopes with/or the added start-up neutron source, such as Cf-252, and a simple but effective method also was introduced and testified for calculating the EFM co-efficiency of arbitrary neutron.

As the detailed geometry of the Godiva-II is not available, the homogenized cylinder core model with the HEU mass and the core outer dimension retained, was used for calculating the EFM co-efficiency with MCNP 5 and ENDF/B VII.1.The EFM co-efficiency of the uranium spontaneous fission source is 0.86 . Since the Caliban reactor has the same shape to the Godiva-II, 0.86 is also suitable for Caliban. Hence the strength of EFM source for Godiva-II and Caliban used for burst waiting time simulation is $58 \mathrm{~s}^{-1}$ and 103 $\mathrm{s}^{-1}$, respectively. 
With the simulation method proposed by Xiao[4], the burst waiting time experiment simulation program are developed, and tested using the same parameter of Godiva-II and Caliban presented in the papers above via comparing the simulation results with the reference. After testing the strength of EFM source were substituted with the values obtained this work and the experiment simulation is recalculated to investigate to what extent the mean waiting time changes. Almost 500 bursts simulation in each round simulation was calculated to reduce the statistics effect. All the results are shown in Table III.

Table III. Results of Burst Waiting Time for Godiva-II and Caliban

\begin{tabular}{|c|c|c|c|}
\hline & $\begin{array}{c}\text { Godiva-II } \\
(\mathrm{s})\end{array}$ & $\begin{array}{c}\text { Caliban } \\
(\mathrm{s})\end{array}$ & Notes \\
\hline Experiment & $3.1 \pm 2.4$ & $0.742 \pm 0.700$ & - \\
\hline Simulation from [4, 5] & $3.2 \pm 2.5$ & $0.808 \pm 0.750$ & Ref. 4 for Godiva-II and Ref. 5 for Caliban \\
\hline Simulation this work, 1 & $2.9 \pm 2.2$ & $0.888 \pm 0.863$ & Same parameters to the 2 ${ }^{\text {nd }}$ entry are used. \\
\hline Simulation this work, 2 & $3.9 \pm 3.5$ & $\mathbf{1 . 7 8 3} \pm \mathbf{1 . 6 9 3}$ & $\begin{array}{c}\text { Same parameters, but the source strength } \\
\text { this work are used. }\end{array}$ \\
\hline
\end{tabular}

From the $3^{\text {rd }}$ entry in Table III, the simulation results this work match well to the results of reference when the same parameters are used in the calculation, also match well to the results of experiment. From the $4^{\text {rd }}$ entry in Table III, if the source strength is replaced with values obtained this work, both the mean burst waiting time, when compared with the experiments, become larger by about $25 \%$ for Godiva-II and 2 times more for Caliban.

The comparisons of waiting time distribution between experiments and simulations for Caliban is shown in Figure. 1. The results of experiment are cited from Ref. 5.

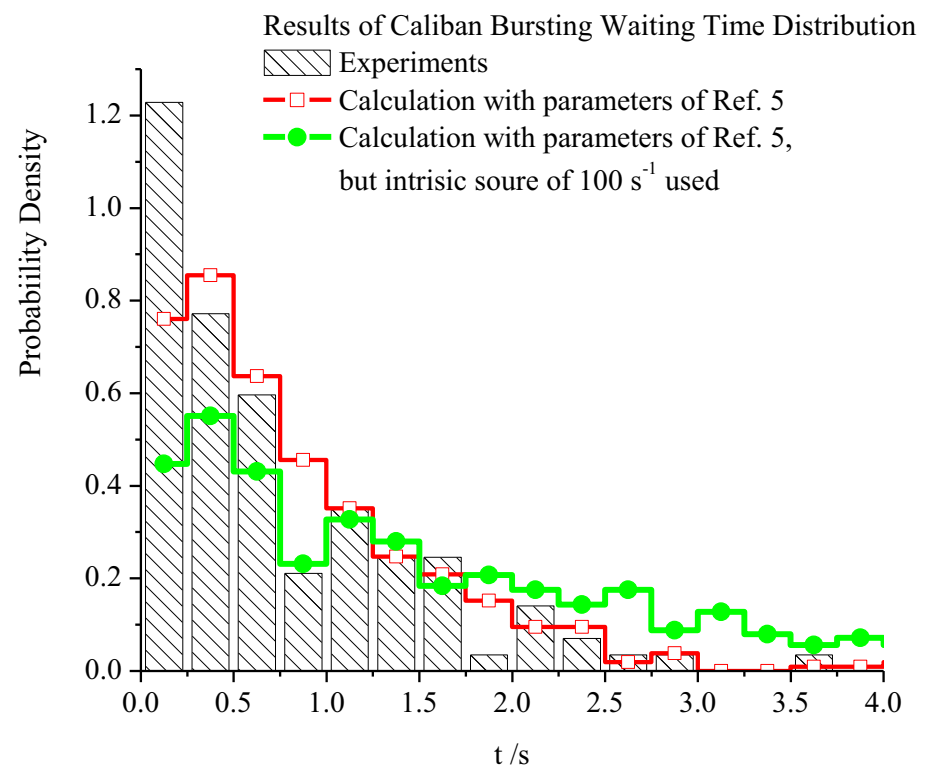

Figure 1. Comparisons between the experiments and simulations 
Obviously, the simulation result of this work for Caliban does not match the experiment results, nor the simulation results presented in Ref.5. This inconsistency most likely implies that the burst waiting time experiment in Caliban had some systematic or undistinguished fault. It is recommended that the experiment configuration need be reviewed again or a new experiment under more rigid procedure and reproducibility need to be carried out to check the inconsistency.

\section{CONCLUSIONS}

The strength of spontaneous fission neutron source for Godiva-II and Caliban, calculated from the SF neutron emission rates of U-235 and U-238, are much less than those proposed by in the reference. When these data are used for burst waiting time simulation, both the mean waiting time become larger by about $25 \%$ for Godiva-II and by 2 times for Caliban than the experiment results. For Caliban the inconsistency between the experiment and simulation results is observed, and more work need to do for clarifying this inconsistency.

\section{REFERENCES}

1 T. F. WIMETT, R. H. WHITE, W. R. STRATTON, et al. Godiva II-An Unmoderated PulseIrradiation Reactor [J]. NUCLEAR SCIENCE AND ENGINEERING, 1960; 8(6): 691-708 2 Philippe Humbert, Nicolas Authier, et al. BURST WAIT TIME SIMULATION OF CALIBAN REACTOR AT DELAYED SUPER-CRITICAL STATE [R], Proc. PHYSOR 2012. Knoxville, Tennessee, 2012, U.S.A.: American Nuclear Society

3 G. E. HANSEN. Assembly of Fissionable Material in the Presence of a Weak Neutron Source [J]. Nucl. Sci. Eng., 1960; 8(6): 709-719

4 X. GANG. The Simulation of Probability Distribution of the Burst Waiting Time of Neutron Initiation [J]. Nucl. Sci. Eng., 2011; 169(1): 56-67

5 Nicolas Authier, Beno1 t Richard. Initiation of Persistent Fission Chains in the Fast Burst Reactor Caliban, NUCLEAR SCIENCE AND ENGINEERING, 2014; 177(2): 169-183

6 Liu Xiaobo, Fan Xiaoqiang, Peng Xianjue, et al. A Novel Experimental Method for Validating Neutron Initiation Probability [J]. Nuclear Science and Engineering, 2015; 179(2): 119-129

7 Liu Xiaobo, Peng Xianjue, Lei Jiarong, et al. Experimental Study of Neutron Initiation Probability at CFBR-II with Pulsed Neutrons [J]. Nuclear Science and Engineering, 2015; 181(1): 96-104

8 Shultis, J. Kenneth, Richard E. Faw. Fundamentals of Nuclear Science and Engineering [M]. ISBN 14200-5135-0,CRC Press, 2008: 141

9 N. Ensslin, W. C. Harker, M. S. Krick, et al. Application Guide to Neutron Multiplicity Counting [R], LA-13422-M. U.S.A.: Los Alamos Scientific Laboratory, 1998

10 G. D. Spriggs, Robert D. Busch. Takeshi Sakurai.et al. The equivalent fundamental-mode source [J]. Annals of Nuclear Energy, 1999; 26(3): 237-264, or Gregory D. Spriggs. The equivalent fundamentalmode source [R], LA-13253. U.S.A.: Los Alamos Scientific Laboratory, 1997

11 U.S. Department of Energy. Nuclear Physics and Reactor Theory, Module 1 - Atomic and Nuclear Physics [M], DOE-HDBK-1019/1-93. U.S.A.: DOE, 1993 